of mental confusion. Chaslin says: "There exists one form of mental disease that is neither mania nor melancholia, and which is due to some abrupt and rapid exhaustion of the nervous system. Caused frequently by infection or auto-intoxication, this condition should be kept separate from forms of degeneration. It occupies a middle ground between psychoses and insanity of definite profound lesions. Often primary mental confusion appears to be a true disease on account of somatic phenomena that accompany it, as malnutrition, fever, etc. Psychically, this state is characterized by confusion of mind. This confusion is due to feebleness and incoördination in the process of association of ideas, in perception, and in personal feeling. It may or may not be accompanied by hallucinations. Muscular excitement may exist, or there may be depression or stupor. The emotional tone is that of indifference or abrupt changes. There is much analogy between primary mental confusion and insanity following chronic intoxications. The term primary is used to distinguish this confusion from similar states that are secondary and of a nature as yet undetermined."

L. F. B.

Alcoholic Insanity.-Dr. S. V. Clevenger, (N. A. Practitioner, March, I892). The writer remarks that chronic alcoholic insanity is rarely seen outside of asylums, where its victims are gathered in considerable numbers.

The delusions of such cases are rather characteristic and consist of delusions of persecution, of having been poisoned, uxiorial unfaithfulness. Mutilation of the genital organs is not uncommon. The physical symptoms of alcoholic insanity, such as tremors, anæsthesia, abnormal sensations, are the same as may be found in ordinary alcoholism.

The great danger to the community is that the alcoholic insane appear at times to be sane. J. C.

\title{
CLINICAL.
}

\section{A Case of Isolated Paralysis of the Serratus} Magnus.-Bruns reports a case of pronounced paralysis of the serratus in whom the most important symptomthe inability to elevate the arm above the horizontal plane-was completely absent.

In the beginning the patient was attacked with severe pain in the entire right arm and shoulder. At first the 
arm could not be moved, which made it difficult to determine whether this absence of motion was due to pain or paralysis. For about six weeks there was actual paralysis of the arm and shoulder. Subsequently, the isolated serratus paralysis became evident. Six months later he still had slight pain in the axilla, but no objective sensory disturbance. Neither galvanic nor faradic reaction could be obtained at the corresponding motor points over the clavicle or at the serrations of the muscle. The other muscles of the arm and shoulder, especially the trapezius, were strong, and gave normal electrical reactions. The upper portion of the trapezius, the region of the infraspinatus and the deltoid were flatter than upon the left side. The patient's arduous labor as a brassfounder was not interfered with. No cause could be discovered indicative of paralysis from neuritis. There were no evidences of lead poisoning. He thus explains the elevation of the arm to the vertical position : at first the deltoid acts in association with the supraspinatus, and probably the infraspinatus, in order to forcibly place the arm at a right angle with the external border of the scapula. For the further elevation, the well-known rotation of the scapula with its point outward must now take place, and this, in a deficiency in the serratus, is accomplished by the middle portion of the trapezius, which at the same time draws the scapula downward.

In this case the external rotation of the scapula could partly be executed by the posterior fibres of the deltoid, and partly by the teres major and minor and the infraspinatus.

It is of interest to note from a clinical standpoint that the most important symptom of serratus paralysis, the one that causes the essential functional disturbance, does not exist in every case. We do not know positively why this should occur in one case and not in another.Neurolog. Centrbl, No. 2, 1893 .

A Case of Hysteria, Associated with Morphine Habit, Cured by Extirpation of Ovaries.-(Dr. Eichholz, in Frauenarzt, Vol. VII., No. I I.) He justifies the operation, when, as in severe cases of hysteria, both physical and psychical impairment is considerable. The patient, aged forty-five, unmarried, had been, since sixteen years of age, under thirty or more physicians' care for a host of diseases. She had been treated at a watercure establishment; had received electrical treatment; had been through a gymnastic training, and also had 